\title{
Particle Swarm Optimization for tuning a Fuzzy Supervisory Controller Parametesrs (Takagi Seguno and Mamdani Engines).
}

\author{
BENDIB Riadl ${ }^{(1)}$, HAMMADI Youcef $^{(1)}$, MAZOUZI Mohammed $^{(1)}$ MECHHOUD Elarkam $^{(2)}$ \\ ${ }^{(1)}$ Department of petrochemical and process engineering. University of Skikda, Skikda, Algeria \\ (2) Department of electrical engineering engineering. University of Skikda, Skikda, Algeria \\ r.bendib@univ-skikda.dz, e.mechhoud@univ-skikda.dz
}

\begin{abstract}
Steam generation systems are a crucial part of most power plants. Therefore, boiler control is an important problem for power plants that are frequently changing load or subject to sudden load disturbances, which are common in current market driven electricity industry. Hence, the control of such processes needs the design of more effective controllers. Although Fuzzy logic controllers seem more adequate for controlling chemical processes, since they provides solutions to incompletely defined and nonlinear processes industrials does not prefer such type of controllers since the tuning of these controllers requires the adjustment of a large number of parameters, which is tedious. The fact that let the industrials prefer the use of simple PID controllers rather than complicated fuzzy or neural controllers. Hence in this paper a simple PD controller is used to control the steam flow parameters of whereas its parameters are deduced using fuzzy supervisory controller the parameters of the supervisory controller are optimized using particle swarm optimization algorithm, the most known inference Engines SEGUNO and MAMDANI are considered.
\end{abstract}

Keywords: Boiler; fuzzy supervisory; PID controller ;particle swarm optimization algorithm

\section{INTRODUCTION}

Boilers were a major part of the Industrial Revolution beginning about 1700 . They are major consumers of industry and building energy consumption today. They are used for power generation, process heat (refineries, petrochemicals, paper mills, tire manufacturing...) and heating [1] The function of a boiler is to deliver steam of a given quality (temperature and pressure) either to a single user, such as a steam turbine, or to a network of many users. Then, a properly functioning boiler must satisfy the following basic requirements:

1) The ratio of air to fuel must be carefully controlled in order to obtain good, safe, and efficient combustion.

2) The level of water in the drum must be controlled at the desired level in order to prevent overheating of drum components or

flooding of steam lines.

3) A desired steam pressure must be maintained at the outlet of the drum despite variations in the quantity of steam demanded by users [2]. The boiler drum is the main component of the boiler. A steam drum is a standard feature of the boiler water pipes. It is a reservoir of water/steam at the upper end of the tube of water. Drums keep the steam produced in the water tubes and act as a phase separator for mixed steam/water. The density difference between hot and cold water helps in the accumulation of -water/and "hot" saturated steam into the steam drum.

The following figure (Fig 1) shows the main component of a typical boiler. The main parameters that affect the operation of boiler is the water flow

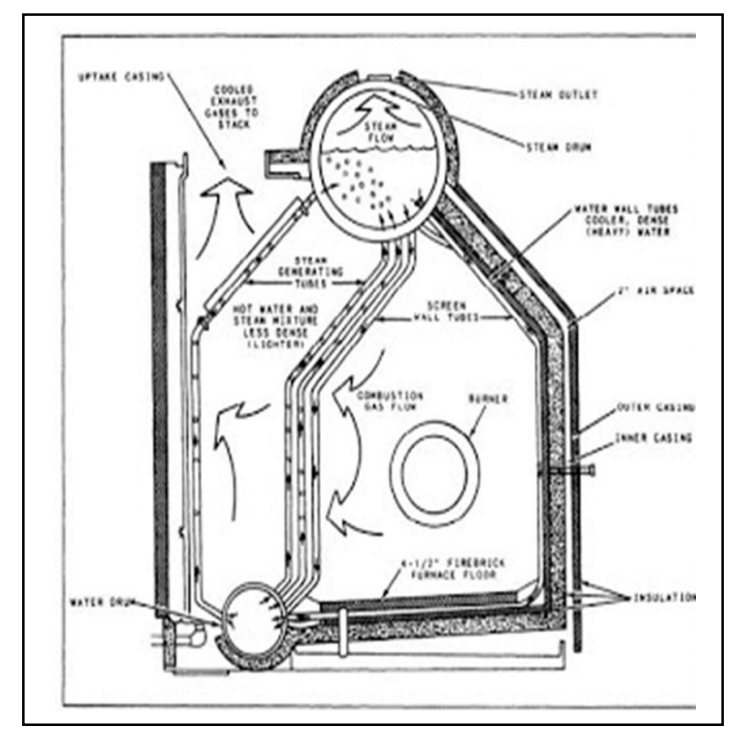

Fig. 1 Schematic picture of an industrial drum boiler 
The performance of a fuzzy logic controller depends on its control rules and membership functions. Hence, it is very important to adjust these parameters to the process to be controlled. A method is presented for tuning fuzzy control parameters by Particle Swarm Optimization to make the fuzzy logic control systems behave as closely as possible to the operator or expert behavior in a control process [3]. In the present paper, we will describe the optimization of membership functions, its parameters and its shapes, in a fuzzy supervisory PD controller for a boiler employing Particle Swarm Optimization tool ( it is one of algorithms that use Artificial intelligence techniques

\section{FUZZY LOGIC}

In recent years, the number and variety of fuzzy logic applications have increased significantly. The applications range from consumer products such as cameras, camcorders, washing machines, and microwave ovens to industrial process control, medical instrumentation, decisionsupport systems, and portfolio selection [4]. Fuzzy logic has two different meanings. In a narrow sense, fuzzy logic is a logical system, which is an extension of multivalued logic. However, in a wider sense fuzzy logic (FL) is almost synonymous with the theory of fuzzy sets, a theory which relates to classes of objects with un-sharp boundaries in which membership is a matter of degree [5]

\subsection{FUZZY LOGIC CONTROL}

Fuzzy Control applications are the kinds of problems for which fuzzy logic has the greatest success and acclaim [6] A fuzzy controller (Fig.2) is a regulating system whose modus operandi is specified with fuzzy rules. In general, it uses a small set of rules. The measurements are processed in their fuzzifier form, fuzzy inferences are computed, and the result is defuzzified, that is, it is transformed back into a specific number [7].

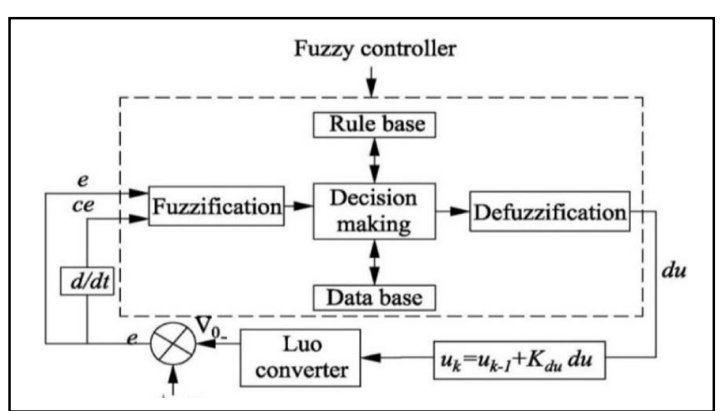

Fig. 2 Block diagram for a fuzzy logic control

\subsection{FUZZY SYPERVISORY CONTROL}

Fuzzy Supervisory controller (Fig.3) is a multilayer (hierarchical) controller with the supervisor at the highest level, as shown in the next Figure. The fuzzy supervisor can use any available data from the control system to characterize the system's current behavior so that it knows how to change the controller and ultimately achieve the desired specifications. In addition, the supervisor can be used to integrate other information into the control decision-making process [8].

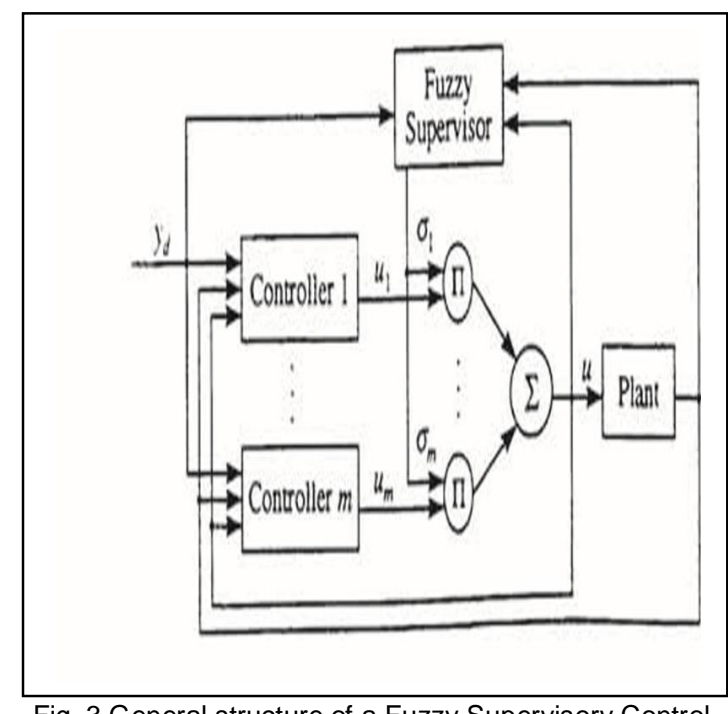

Fig. 3 General structure of a Fuzzy Supervisory Control

\section{PARTICLESWARM OPTIMIZATION}

Particle swarm optimization (PSO) is a computation technique first proposed in 1995 by Kennedy and Eberhart $[9,10]$. This method has been found to be a robust method in solving non-linearity or non-differentiability problems, PSO algorithm didn't use evolutionary operators (mutation or crossover to manipulate algorithms). However, it simulates a dynamically population behavior (fish swarm or bird flocks), where social sharing of information takes place and individuals can profit from the discoveries and previous experience of all the other companions during the search for food. Thus, each companion is called particle and the population is called swarm it is assumed to fly in many directions over the search space in order to meet the demand fitness function $[11,12,13]$. For $n$-variables optimization problem, a flock of particles are put into the n-dimensional search space with randomly chosen velocities and positions knowing their 
best values, so far (Pbest) and the best position in the $\mathrm{n}$-dimensional space. The velocity of each particle, adjusted accordingly to its own flying experience and the other particles flying experience. For the itch particle and n-dimensional space can be represented as equation (6), the best previous position of its particle is recorded as equation (2):

$\mathrm{x}_{\mathrm{i}}=\left(x_{i, 1}, x_{i, 2}, \ldots \ldots \ldots x_{i}\right)$

$p_{\text {best }}=\left(p_{\text {besti,1 }}, p_{\text {besti,2 }} \ldots\right.$ pbesti,n $\left._{\text {bes }}\right)$

The velocity is an essential part of how PSO work [14] so as modified velocity and position of each particle can be calculated using the current velocity and distance from ( $p$ besti,) to

$v_{i, m}^{(I t+1)}=w * v_{i, m}^{(I t)}+c 1 R 1\left(p_{\text {best }}-x_{i, m}^{(I t)}\right)+c 2 R 2\left(g_{\text {best }}-x_{i, m}^{(I t)}\right)$

$x_{i, m}^{(I t+1)}=x_{i, m}^{(I t)}+v_{i, m}^{(I t)}$

(gbestd) as shown in equations. $(3,4)$

Where:

$\mathrm{i}=1,2$, Number of particles.

$\mathrm{m}=1,2, \ldots$. Dimension.

It.: Iterations pointer.

『 $v{ }_{-}(\mathrm{i}, \mathrm{m})^{\wedge}((\mathrm{it}))$ : Velocity of particle $\mathrm{i}$ at iteration (it)

W:weight factor.

c1,c2: Acceleration constant.

$r$ : Random number between (0-1).

$\mathrm{x} \_(\mathrm{i}, \mathrm{m})^{\wedge}((\mathrm{it}))$ : Current position of particle i at iteration It.

pbesti: Best previous position of ith particle.

gbestm: Global best particle among all the particles in the population.

The following flowchart (Fig.4) shows an algorithmic description for the computer implementation of particle swarm optimization algorithm..

The first step, is the specification of initial particles where the position and velocity are random, after that and similar to other heuristics methods this position is evaluated by considering a fitness function, after that a new position and velocity are calculated and the algorithm is updated after defining the global best is calculated and in each iteration a stop criteria is tested in case the convergence is achieved so the stop otherwise we will repeat the same procedure till the convergence.

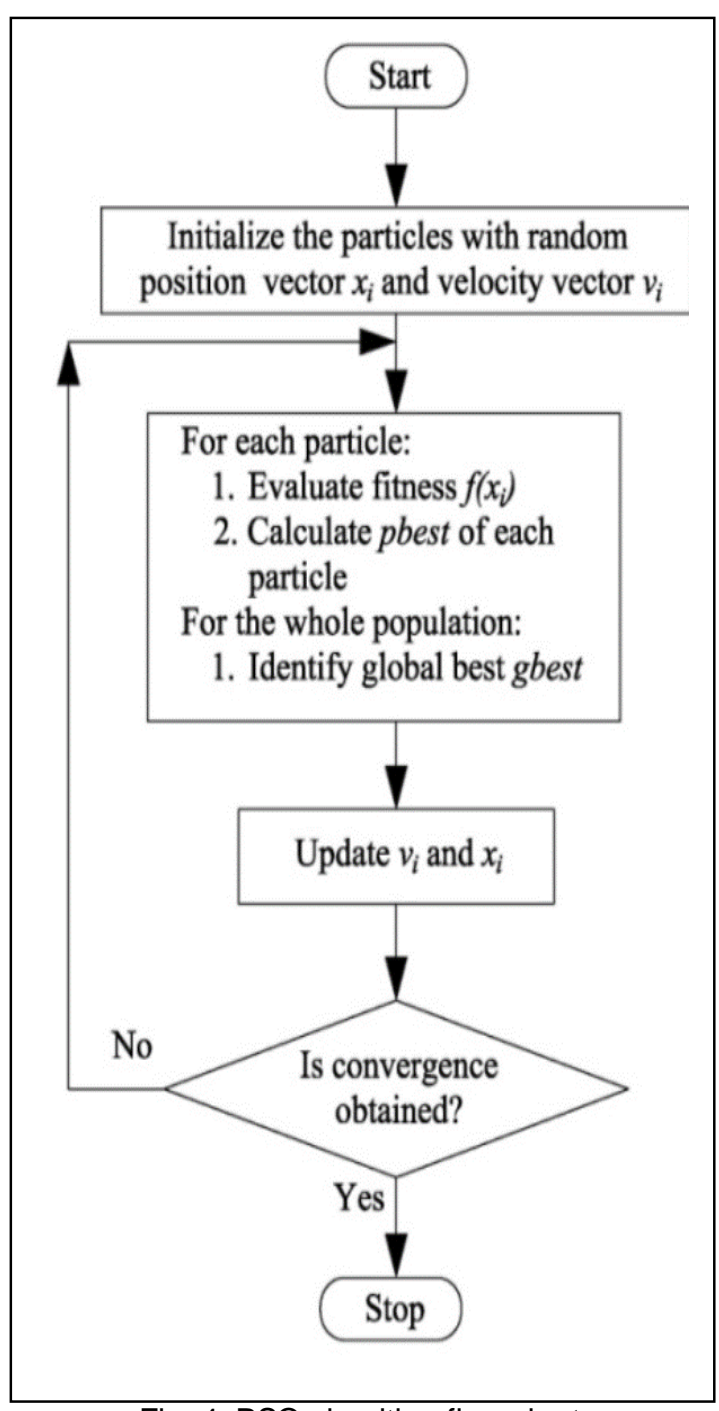

Fig. 4. PSO algorithm flow chart

\section{POCESS DESCRIPTION}

A boiler of a chemical plant is taken as a case study and the temperature control of the boiler is controlled using fuzzy supervisory controller the real input of controlling the temperature is controlling the flow of steam in a heat exchanger. The Fuzzy controller parameters such as the memberships function shape and rule base are optimized using PSO algorithm as it is mentioned in the following flow chart Fig.5

The boiler is mathematically modeled using experimental data available and its transfer function is given as [15]:

$$
G(S)=\frac{5(s+1)}{s(s+6)(s+1)}
$$




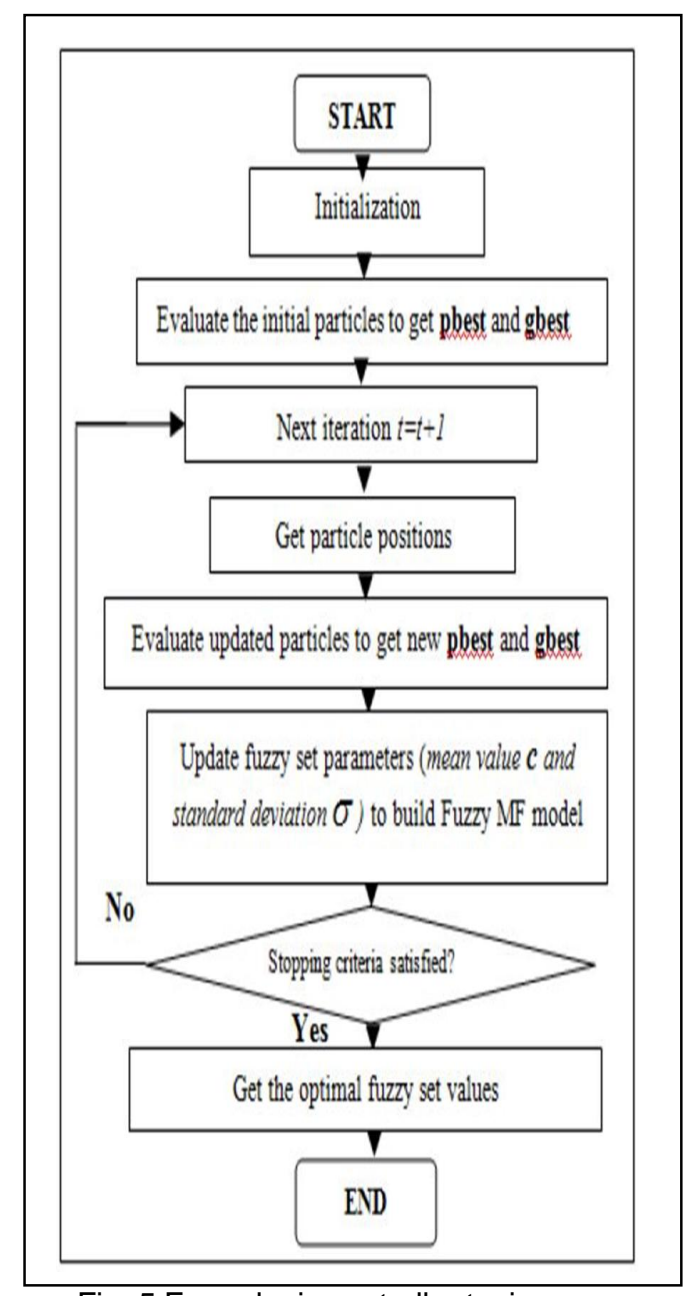

Fig. 5 Fuzzy logic controller tuning process

The algorithm works based on determination of the best shape and rule that satisfied the requirement of a fitness function that is constructed based on the steady state error. For Mamdani inference engine the basic shapes for inputs and ouputs are trapezoidal shapes .Concerning TS the shapes for the inputs are also trapezoidal,

\section{SIMULATION AND RESULTS}

The objective in this paper is to minimize the error which represents the difference between the response of our system and the set point. Hence the fitness function is defined as follows:

$$
J=\sum_{i=1}^{k} e i^{2}
$$

The fuzzy logic supervisory has two input values (error and delta-error) each one of them consisted of five trapezoid membership function equally distributed. The two outputs are the PD controller parameters $\mathrm{kp}$ and the $\mathrm{Kd}$ consisted also of three MFs also equally distributed in the case of Mamdani they are trapezoid function but in Sugeno are constant. In the tuning process with the particle swarm optimization, we tuned just the shape of the function with fixing the number of MFs and the rules. For this process we make each trapmf sweep just a part of the range to gain time and computation power. But we fix some point to allow each function to change the shared parts between the function. The PSO parameters are chosen as indicated in Table 1.

Table 1 Table caption

\begin{tabular}{|l|c|}
\hline The number of populations & 20 \\
\hline The number of iterations & 30 \\
\hline The self-adjustment factor & Chi*Phil \\
\hline The social-adjustment factor & Chi*Phi2 \\
\hline Inertia weight & chi \\
\hline Selection & Ring \\
\hline
\end{tabular}

Where

Kappa $=1$;

Phi1 = 2.05;

Phi2 = 2.05;

Phi $=$ phi1 + phi2 ;

Chi $=2^{*}$ kappa/abs(2-phi-sqrt(phi^2-4*phi))

Moreover, the fuzzy logic parameters are summarized as follow

$$
\begin{gathered}
\text { * } E[-0.01,0.01] \text { AND } \Delta E \in[- \\
0.1,0.1] ;
\end{gathered}
$$

* The range of "KP" is [10;16] and "KD" [1.5;3.5].

Minimum inference engine (Mamdani inference \& Sugeno inference)

* The rules are in table 2

Table 2 Fuzzy rules for $\mathrm{Kp}$ and $\mathrm{Kd}$

\begin{tabular}{|c|c|c|c|c|c|}
\hline $\begin{array}{c}\Delta \mathbf{e} / \\
\mathbf{E}\end{array}$ & $\mathbf{N G}$ & $\mathbf{N}$ & $\mathbf{Z}$ & $\mathbf{P}$ & $\mathbf{P G}$ \\
$\mathbf{N G}$ & $\mathrm{N}$ & $\mathrm{N}$ & $\mathrm{N}$ & $\mathrm{P}$ & $\mathrm{P}$ \\
\hline $\mathbf{N}$ & $\mathrm{N}$ & $\mathrm{N}$ & $\mathrm{N}$ & $\mathrm{Z}$ & $\mathrm{P}$ \\
\hline $\mathbf{Z}$ & $\mathrm{N}$ & $\mathrm{N}$ & $\mathrm{Z}$ & $\mathrm{P}$ & $\mathrm{P}$ \\
\hline $\mathbf{P}$ & $\mathrm{N}$ & $\mathrm{Z}$ & $\mathrm{P}$ & $\mathrm{P}$ & $\mathrm{P}$ \\
\hline $\mathbf{P G}$ & $\mathrm{Z}$ & $\mathrm{P}$ & $\mathrm{P}$ & $\mathrm{P}$ & $\mathrm{P}$ \\
\hline
\end{tabular}




\section{- Case 1 MAMDANI inference engine}

The optimum number and shape of the fuzzy inputs and outputs are shown with Mamdani inference in Fig.6

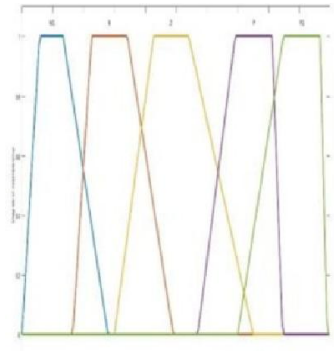

a) error

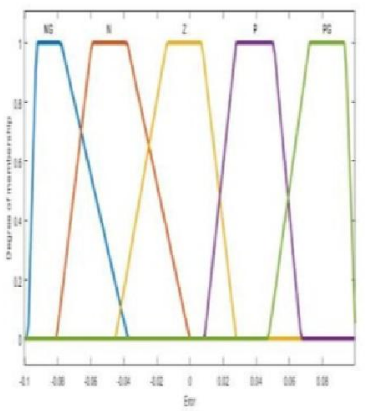

b) delta error
Fig. 6 the optimum mumbership functions .

The optimum number and membership functions for the the fuzzy controller output ( $\mathrm{Kp}$ and $\mathrm{Kd}$ ) following MAMDANI are indicated in Fig. 7.

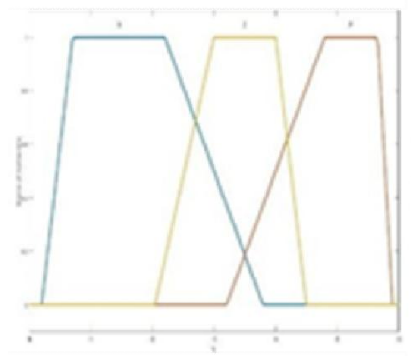

$\mathrm{a}-\mathrm{kp}$

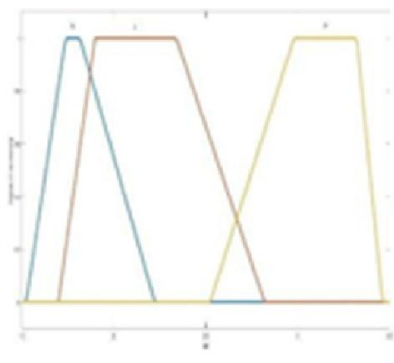

$b-k d$
Fig. 7 the optimum mumbership functions $\mathrm{Kp}$ and $\mathrm{Kd}$

The responses that correspond the obtained optimum fuzzy supervisory PD controller are shown in the Fig 8. The most desirable performance requires the controller to have the smallest possible value for the rise time and the settling time are investigated

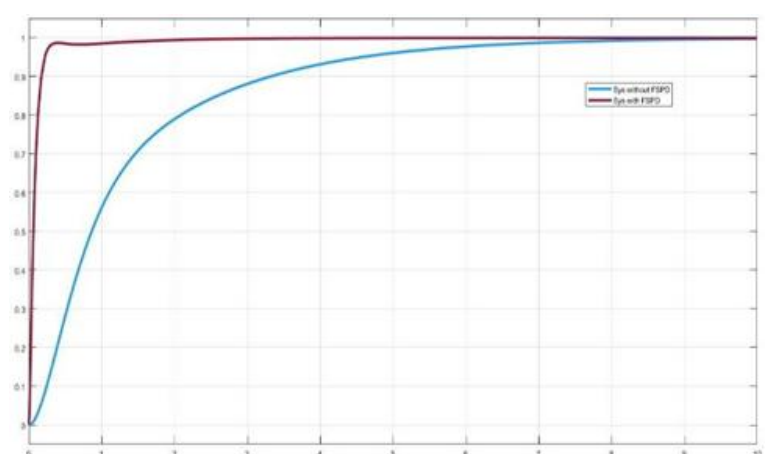

Fig. 8 the corresponding time responses Mamdani

\section{- Case 2 TAKAGI SEGUNO engine}

The resulting optimum inputs membership functions trapezoidal shape Fig 9.
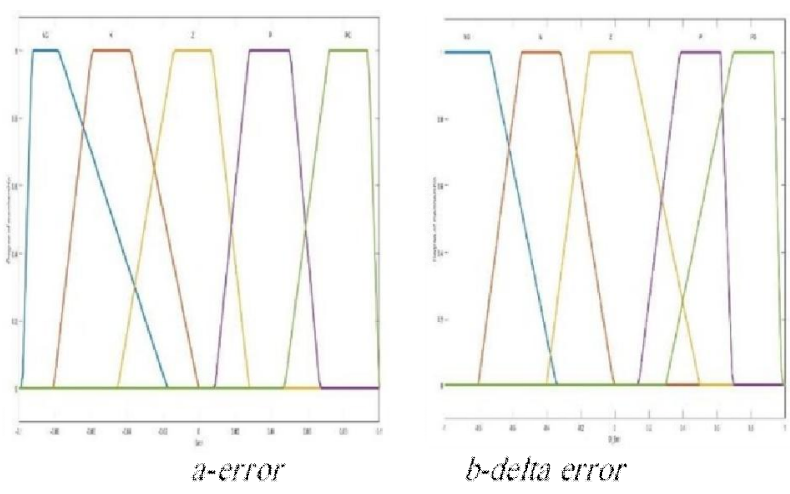

Fig. 9 the optimum mumbership functions

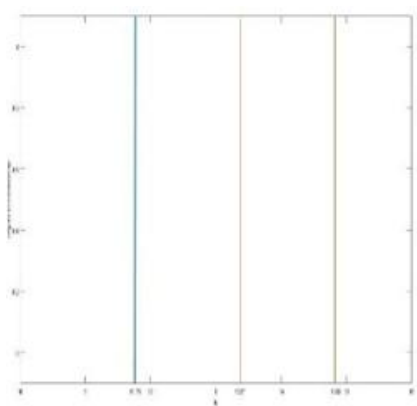

$\mathrm{a}-\mathrm{Kp}$

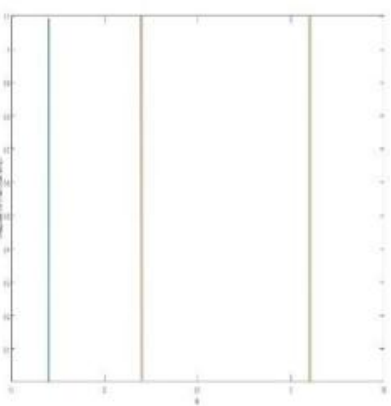

b-Kd
Fig10. The optimum membership functions The responses that correspond the obtained optimum fuzzy supervisory PD controller are shown in the Fig 11. The most desirable performance requires the controller to have the smallest possible value for the rise time and the settling time are investigated

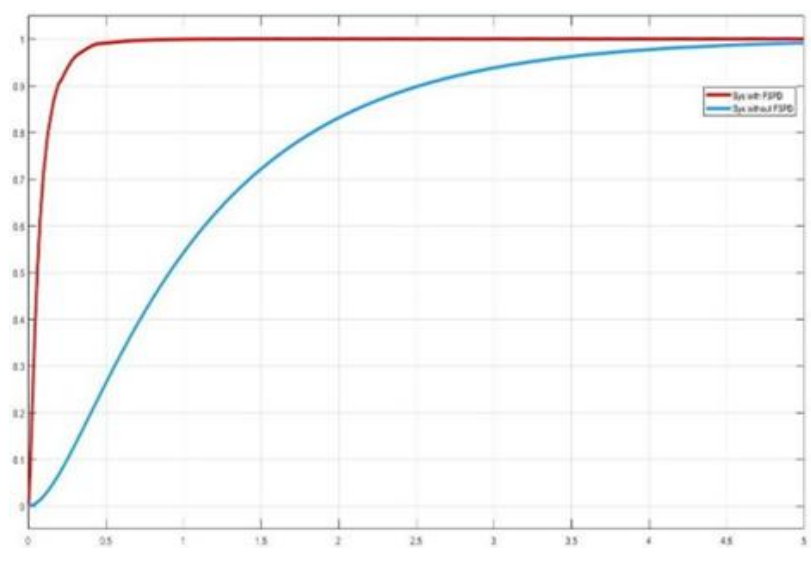

Fig. 11 the corresponding time response 


\section{CONCLUSION}

The simulation results demonstrate the efficiency of Particle swarm optimization Algorithms to be powerful search tools that can reduce the time and effort involved in designing controllers. They can quickly find close to optimal solutions and if set-up well can avoid local optimal. Also, we could observe that Sugeno inference is much better result and faster time in defining a good result because it doesn't require tuning the shapes of the output function (we estimated about 30 $\%$ gain in time comparing to Mamdani's inference).

\section{References}

[1] W.Tan, J.Lin, Y.Chan, " Tuning og PID controllers for boiler-turbine units", 2004.

[2] F.Morilla, "Benchmark for PID control based on the boiler control problem", IFAC Conference on advances in PID control, Brescla(Italy), 2012.

[3] zung-Pei Hong, Chai-Ying Lee, "Introduction of fuzzy rules and membership functions from training examples", from "Fuzzy Sets and System 84 page 33-47.", National Science Council of the republic of China ,(1995-1996).

[4] Li-Xin Wang ,'A course in fuzzy systems and control", International Edition 'Prentice Hall International, Inc' (1997).

[5] Kevin M. Passino -Department of Electrical Engineering The Ohio State University-, Stephen Yurkovich -Department of Electrical Engineering The Ohio State University- ,"Fuzzy Control", An Imprint of Addison- Wesley Longman, Inc;(1998). [6] K. Timothy J.Ross ,"Fuzzy Logic With Engineering Applications", "University of New Mexico,USA', WILEY 'A John Wiley and Sons, Ltd., Publication' ;third edition 2010.

[7] R. Rojas,"Neural Networks", Springer-Verlag, Berlin (1996).

[8] Frank Dernoncourt, "Introduction to fuzzy logic", MIT, (January 2013).

[9] Chen, A.-L., Yang, G.-K., Wu, Z.-M.: Hybrid discrete particle swarm optimization algorithm for capacitated vehicle routing problem. Journal of Zhejiang University Science, 607-614 (2006).

[10] Zhou, C., Gao, L., Gao, H.-B., Zan, K.: Particle Swarm Optimization for Simultaneous Optimization of Design and Machining Tolerances. In: Wang, T.-D., Li, X., Chen, S.-H., Wang, X., Abbass, H.A., Iba, H., Chen, G.-L., Yao, X. (eds.) SEAL 2006. LNCS, vol. 4247, pp. 873-880. Springer, Heidelberg (2006).

[11] Xie, X.-F., Zhang, W.-J., Yang, Z.-L.: Adaptive Particle Swarm Optimization on Individual Level. In: International Conference on Signal Processing (ICSP), Beijing, China, pp. 1215-1218. IEEE (2002).

[12] Esmin, A.A.A., Lambert-Torres, G., de Souza, A.C.Z.: A Hybrid Particle Swarm Optimization Applied to Loss Power Minimization. IEEE Trans. on Power Systems 20(2), 859-866 (2005).
[13] Lin, W.-M., Hong, C.-M.: A New Elman Neural Network Based Control Algorithm for Adjustable Pitch Variable Speed Wind Energy Conversion Systems. IEEE Trans. On Power Electronics 26(2), 473-481 (2011).

[14] Pedersen, M.E.H.: Tuning \& Simplifying Heuristical Optimization Thesis for the degree of Doctor of Philosophy submitted to University of Southampton Computational Engineering and Design Group School of Engineering Sciences (January 2010).

[15] Rahul Malhotra1, Rajinder Sodhi "Boiler Flow control using PID 